\title{
Improving the Patient Discharge Planning Process through Knowledge Management by Using the Internet of Things
}

\author{
Nitya Ahilandam Kamalanathan, Alan Eardley, Caroline Chibelushi, Tim Collins \\ Faculty of Computing Engineering and Sciences, Staffordshire University, Stafford, UK \\ Email: N.A.Kamalanathan@staffs.ac.uk
}

Received April 12, 2013; revised May 20, 2013; accepted May 27, 2013

Copyright (C) 2013 Nitya Ahilandam Kamalanathan et al. This is an open access article distributed under the Creative Commons Attribution License, which permits unrestricted use, distribution, and reproduction in any medium, provided the original work is properly cited.

\begin{abstract}
The UK National Health Service (NHS) is faced with problems of managing patient discharge and preventing the problems that result from it such as frequent readmissions, delayed discharge, long waiting lists, bed blocking and other such consequences. The problem is exacerbated by the growth in size, complexity and the number of chronic diseases in the NHS. In addition, there is an increase in demand for high quality care, processes and planning. Effective Discharge Planning (DP) requires practitioners to have appropriate, patient personalised and updated knowledge in order to be able to make informed and holistic decisions about a patients' discharge. This paper examines the role of Knowledge Management $(\mathrm{KM})$ in both sharing knowledge and using tacit knowledge to create appropriate patient discharge pathways. The paper details the factors resulting in inadequate DP, and demonstrates the use of Internet of Things (IoT) and Machine2Machine (M2M) as candidate technologies and possible solutions which can help reduce the problem. The use of devices that a patient can take home and devices which are perused in the hospital generate information, which can serve useful when presented to the right person at the right time, thus harvesting knowledge. The knowledge when fed back can support practitioners in making holistic decisions with regards to a patients' discharge.
\end{abstract}

Keywords: National Health Service (NHS); Knowledge Management (KM); Discharge Planning (DP); Internet of Things (IoT); Machine2Machine (M2M)

\section{Introduction}

The NHS, a publicly funded organisation, provides healthcare for all UK citizens (currently more than 62 million people) [1]. The NHS is divided into primary and secondary care [1]. Patients requiring further attention are usually transferred from Primary care (PC) to Secondary care (SC). Both PC and SC have links between one another and cannot exist without the other [1]. The sharing of information about a patient between a PC and SC is therefore important. The NHS, like any other healthcare system and other systems, is made up of subsystems which have conventional components such as inputs, processes and outputs. These systems and subsystems are interdependent and inter-related. It is therefore important to understand healthcare subsystems in order to gain a deeper insight into the functioning of the system $[2,3]$. The research project that this paper describes therefore focuses on analysing the hospital system in terms of its structure and process in terms of:
- The components themselves (e.g. patients, nurses) and their roles in the system;

- The relationship between the components and their interaction (e.g. nurses care for patients);

- The boundaries of the system or its extent and scope (e.g. where an admission ward hands over to an operating theatre) or where patients are discharged;

- How the system deals with and adapts to changes within the organisation (e.g. emergency admission or an outbreak of an infection);

- How the system deals with its internal factors (e.g. changes in management, targets, IT systems etc.);

- The relationship of the system to external systems whose services are vital to a patients convalescence (e.g. social care systems);

- The knowledge flow within the system and subsystems.

With sound understanding of the system and subsystems, the practitioner is able to understand the knowledge required, the knowledge which currently exists and can 
be updated to make informed decisions in processes such as DP. Patient discharge can be considered to be the beginning of convalescence. Careful planning of post-treatment care is essential to a patient's complete healthcare pathway, which is an essential component of DP. Careful planning and a clear decision-making framework are vital to the smooth flow of patients from admission to discharge at the end of the treatment period. The NHS has grown, since it was launched in 1948 and is continuously growing in size and complexity [1]. This growth in size, complexity and the number of chronic diseases (e.g. obesity, diabetes) in the NHS causes an increase in demands, processes and planning [1]. A consequence of the complexity, increasing size and demands on the NHS is a disarray of processes that affect functions such as DP. DP is a key part of the overall process and is not an isolated or final event [1]. It is important to include what happens to a patient after discharge, to prevent unwanted readmissions, delayed discharged, bed blocking, cancellations in procedures and long waiting lists. It has implications for the provision of resources in the healthcare, social care and other support service sectors and warrants this research to improve its efficiency and effectiveness. A smooth DP process facilitates patients moving from one healthcare setting to another, or going home. It begins on admission and is a multidisciplinary process involving physicians, nurses, social workers, and possibly other health professionals [4]. The aim of DP is therefore to enhance the continuity of care and can have significant implications for a patient's wellbeing and recovery, the efficient use of medical resources and streamlined interconnecting processes within the hospital setting.

The complexity of the discharge process implies that careful planning is needed to make it more effective [5]. Recent years have witnessed significant advances in medical informatics to increase productivity and efficiency in healthcare [6]. Some parts of the NHS are currently faced with the problem of "islands of information"1 related to the existence of organisational "silos"2. In some cases, it is suggested that very little knowledge is shared between these silos. This leads to the foundation of this paper, which is to examine the role of $\mathrm{KM}$ in an integrated "cross-silo" approach to use shared knowledge and tacit knowledge to create appropriate patient discharge pathways. The tacit knowledge of Doctors and Nurses is yet to be exploited to its full potential along with the knowledge of patients and carers. According to [7] patients, carers and information are the most underutilised resources the NHS has. It is the patients who are

\footnotetext{
${ }^{1}$ IT applications that were originally developed to solve localised problems, but which do not communicate with other applications in the same IT infrastructure.

${ }^{2}$ Parts of the organisation (e.g. departments, functions) that are separate in terms of processes, communication and policies.
}

facing the symptom, and the carers who look after the patient, the knowledge they have is most valuable to making decisions, which unfortunately is currently overlooked. Each patient has a unique problem and personalising the discharge process will reduce the current problems faced with DP. KM therefore forms a bridge between these "islands of information" [8].

\section{The Current Discharge Planning Dilemma in the NHS}

Discharge is defined when an in-patient leaves an acute hospital to return home, or is transferred to a rehabilitation facility or an after-care nursing centre [9]. DP should commence as early as possible in order to facilitate a smooth discharge process. Discharge guidelines have been prescribed by the Department of Health (DH) and different trusts implement discharge pathways or process maps following these guidelines. Several DP improvement attempts have been made and reasonable improvements have been noticed. Several methods by which DP takes place have been identified in the primary research in two UK hospital trusts and include the following:

- DP commences on admission;

- Patient and carer are involved in the decision making process;

- A clinical management plan where an expected date of discharge is predicted based on actual performance in the ward or, on benchmarking information from past cases;

- Multidisciplinary teams make a decision based on experience during their meetings.

A bed management system stores information on beds occupied and a weekly meeting are held to decide the discharge date for patients. All of these methods involve KM. From the Primary research carried out, it is seen that, a rough DP is currently drafted for patients upon entry to hospital according to their diagnosis, and a tentative discharge date is provided in line with recommendations. Changes are made over the course of the patient's stay and records are manually updated by nurses, upon instruction by the doctors. This sometimes results in confusion and even disagreement on discharge dates by different doctors (e.g. when treating the patient for different symptoms) and nurses (e.g. when a change of shift occurs). This research proposes that Patient DP requires viewing the whole system and not as isolated units. In the discharge plan the patient and care giver involvement needs to be considered, however very little indication has been provided on these. To date, based on the primary research, clear guidelines are not present on what information needs to be collected, stored and reused on patients. The UK NHS is facing problems of managing patient discharges while having to meet waiting time, treatment time and bed usage targets [10]. Patient dis- 
charge is currently being driven by quantitative measures such as targets (e.g. to reduce "bed-blocking") and problems resulting from this situation has received a great deal of popular press attention recently and political capital has been made from this [10]. Targets are prioritized while compromising patient's after-care quality.

Being target-driven (rather than knowledge driven) implies that the healthcare system fails to consider the factors that affect the effective recovery of a patient after treatment and discharge [11]. Hospitals focus on accomplishing and achieving internal targets, resulting in compromised patient safety and well-being after discharge. The exact situation with regard to patient discharge and readmissions is not really well established, as there are variations in discharge methods between trusts, as identified in the primary research. However, it is reported in the popular press that doctors have to make quick decisions about patients just to "get the clock to stop ticking" [12] resulting in deteriorating trust between doctors and patients. More reliably, doctors find themselves torn between meeting targets and providing their sick patients with the best treatment. These claims in the assorted news media have been reaffirmed by Andrew Lansley the Secretary of State for Health in the UK Government who in a speech in December 2011 stated that:

"The NHS is full of processes and targets, of performance-management and tariffs, originally, all designed to deliver better patient care, but somewhere along the line, they gained a momentum of their own, increasingly divorced from the patients who should have been at their centre."

(Guardian 7 December 2012)

Several factors result in the current inadequate DP. These factors are internal and external to the NHS along with psychosocial factors of the patient and family [13]. It is important to understand the factors behind inadequate DP to be able to analyse and diagnose the factors causing the problem systematically. A comparison can then be made between the factors along with the results obtained from the primary research, followed by a catalogue of possible solutions underpinned by KM. This will then lead to making a diagnosis i.e. the proposed KM model. A root cause analysis [14] highlighted the factors contributing to inadequate DP as represented in Figure 1.

Figure 1 demonstrates the discharge of a patient as a complex process, with various inter-related factors. A

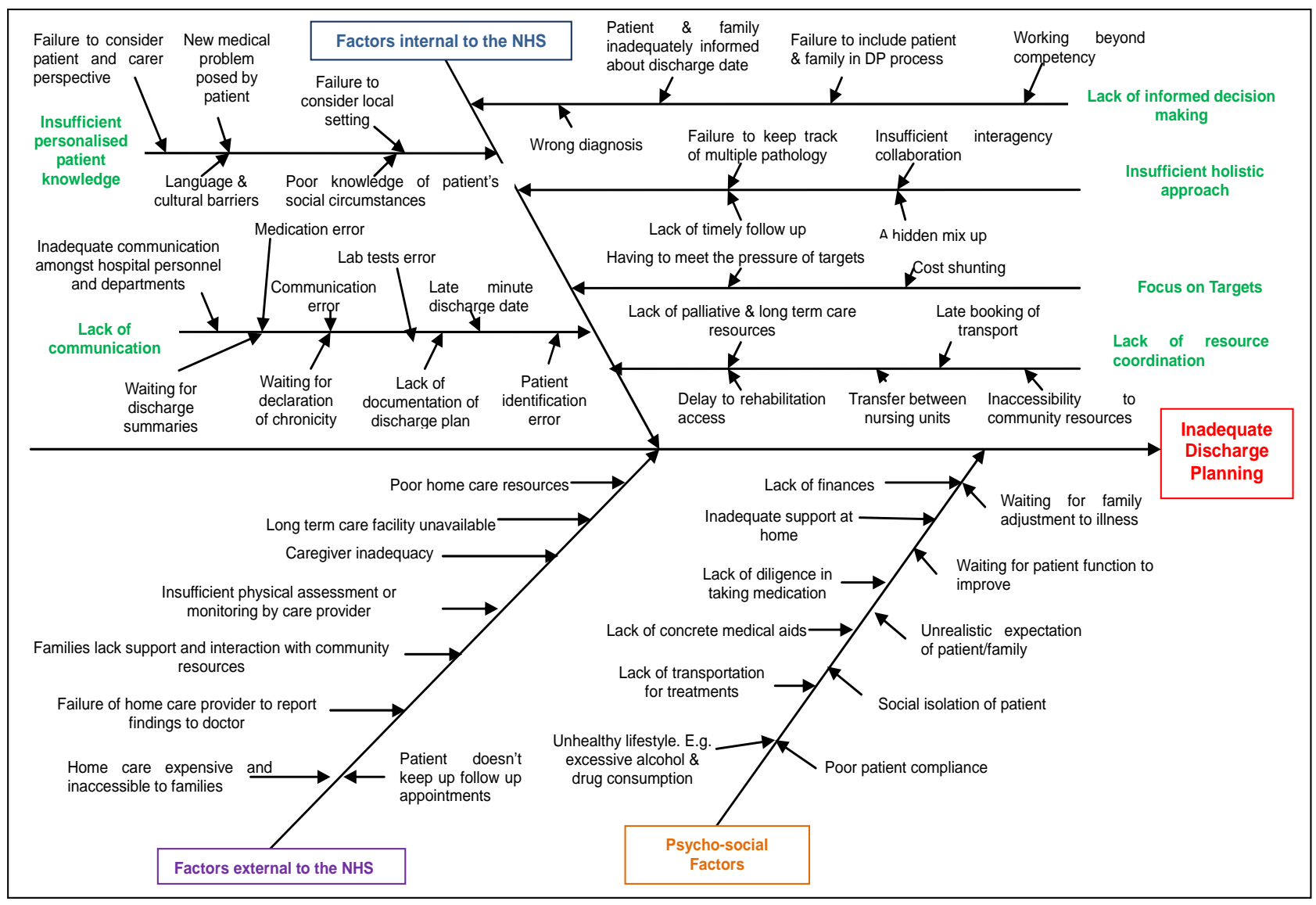

Figure 1. Root Cause Analysis of factors resulting in inadequate DP [12]. 
carefully designed discharge plan supported by KM can ensure more efficient utilisation of hospital resources and will encourage better inter-department communication to ensure that tacit knowledge makes more informed decisions about patient discharge. It is believed that this in turn will allow for better coordination of the external factors and will give hospital personnel more time to inform patients and their families, thus addressing the psychosocial factors. At discharge, preventable and undetected errors can occur. These can be reduced by knowledge sharing among hospital staff and having patient centric discharge pathway leading to improved DP. Patient participation and understanding in discharge process will help reduce potential readmissions and delayed discharge. Patient participation in the discharge process is a legally stated right in the United Kingdom and therefore more active participation of patients is encouraged [15]. The failure to assess a patient's care needs correctly can result in a disproportionate delay in patients being discharged [16]. The problems caused by inadequate DP have been identified in the secondary and primary research and are summarized succinctly in Figure 2.

The number of patients readmitted to hospitals through Accident and Emergency (A\&E) departments within 28 days of being discharged has risen steadily from 359,719 in 1998 to 546,354 in 2008 [17]. While in 2010 more than 660,000 patients were re-admitted to hospital within 28 days of discharge [18]. According to statistics provided by the Department of Health, in England in 20102011 the total number of patients who were readmitted was 561,291. According to the statistics, readmission rates in England have been rising since 2001-2002 to 2010-2011. Figure 3 follows the increasing trend of the percentage of patients readmitted for treatment to UK acute hospitals within 30 days of discharge and a "line of best fit" shows the regularity (and therefore the predictability) of the rise.

The problem of inadequate DP does not just concern readmissions, however. "Bed-blocking" due to delayed discharge has equivalent negative implications. It is reported by the NHS confederation that one in four patients are occupying beds when they could be recovering at home [16], which results in longer waiting lists, loss of confidence in the NHS and escalating expenditure. The average number of patients and days of delayed discharge per month in England for the year 2012 according to the Department of Health was 3997 patients and 114,386 days respectively.

\begin{tabular}{|c|c|c|c|c|c|}
\hline $\begin{array}{c}\text { Insufficient } \\
\text { personalised } \\
\text { patient knowledge }\end{array}$ & $\begin{array}{c}\text { Lack of } \\
\text { informed } \\
\text { decision making }\end{array}$ & $\begin{array}{c}\text { Focus } \\
\text { on } \\
\text { targets }\end{array}$ & $\begin{array}{c}\text { Insufficient } \\
\text { holistic } \\
\text { approach }\end{array}$ & $\begin{array}{c}\text { Lack of } \\
\text { resource } \\
\text { coordination }\end{array}$ & $\begin{array}{c}\text { Lack of } \\
\text { communication }\end{array}$ \\
\hline
\end{tabular}

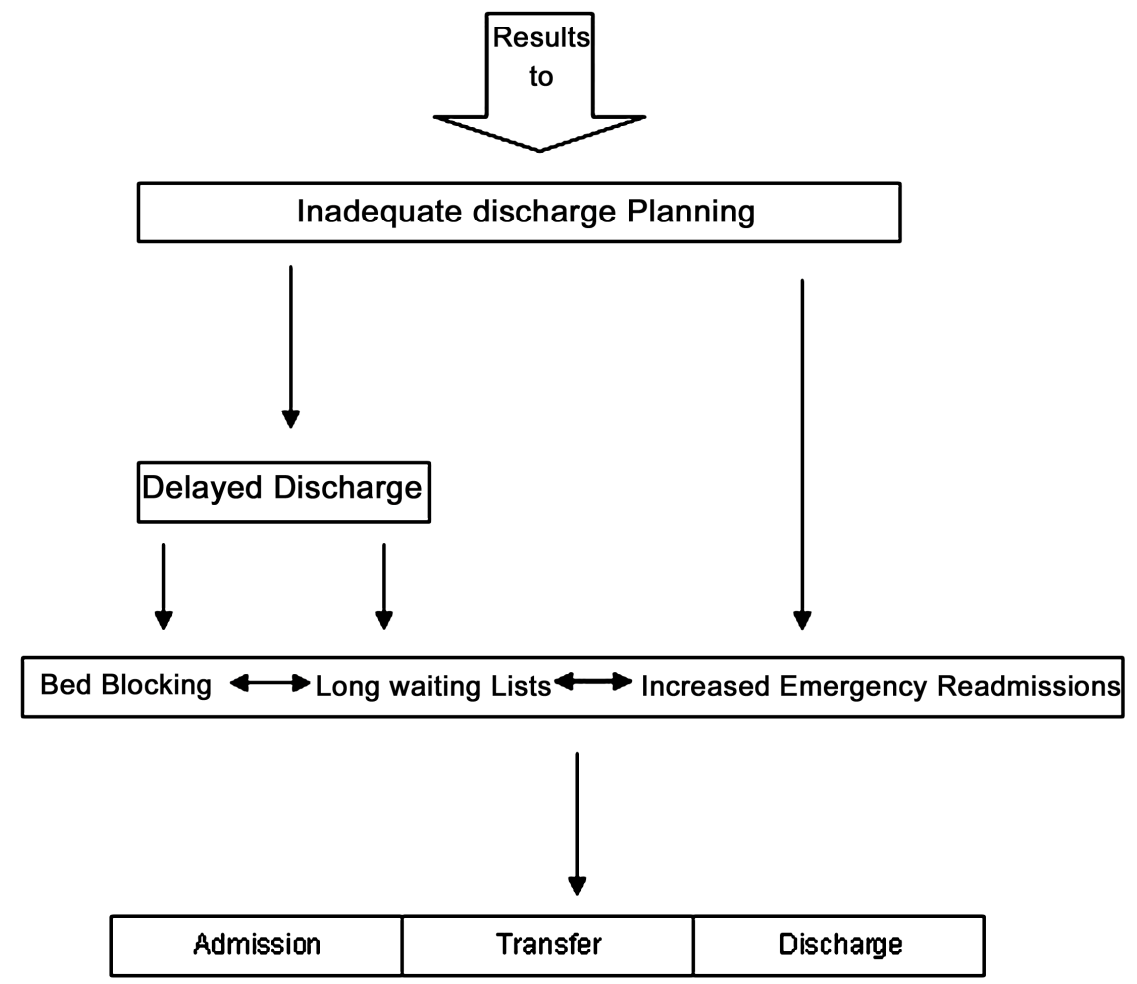

Figure 2. Problems resulting from inadequate discharge planning [12]. 


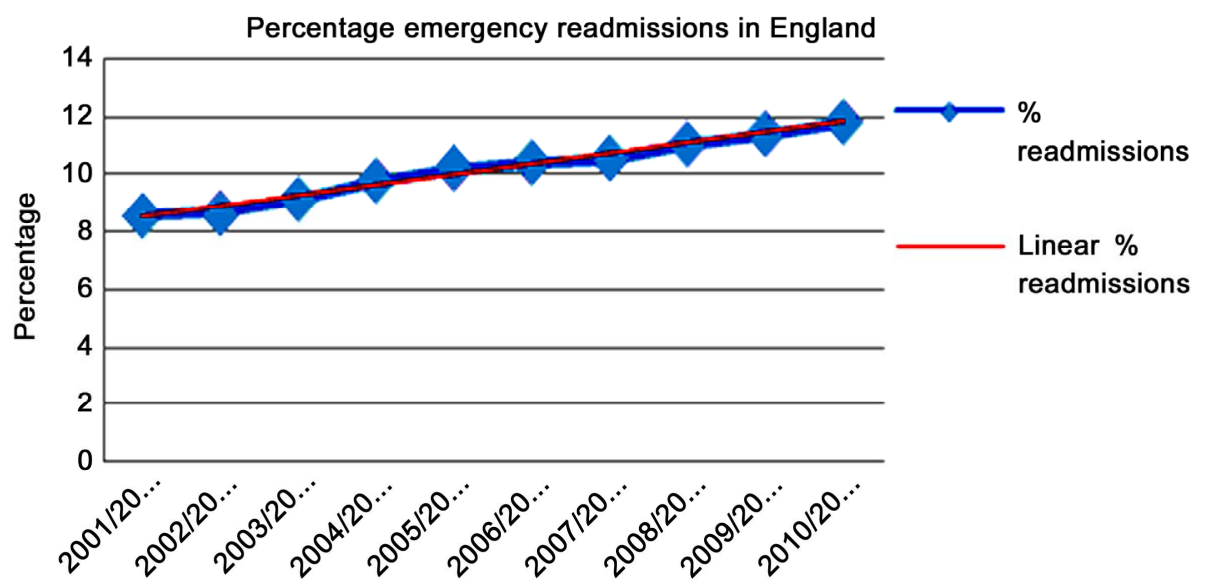

Figure 3. Emergency readmissions in England as a percentage of admissions [12].

Approximately $£ 250 \mathrm{~m}$ was spent on "delayed discharges" between August 2010 and the end of 2011, amounting to $£ 550,000$ a day [17]. Apart from the financial implications the delay in discharge is clearly disadvantageous to the well-being of patients, the morale of their relatives and wastes valuable hospital resources. The King's Fund reports that if it was better organised the NHS could reduce the number of overnight stays by 2.3 million, freeing up 7000 beds and saving the NHS nearly $£ 500 \mathrm{~m}$ a year [18]. Mike Farrar, the Chief Executive of the NHS Confederation indicates that these problems are the result of an "outdated hospital model of care" [19] while a breakdown in communication may also be a possible contributory cause [17]. Many older patients face the brunt of delayed discharge [12] as due to a lack of communication between the NHS and social care homes, they are forced to stay in hospital, causing longer waiting lists for other patients who are seeking urgent treatment [20]. The reasons for the dilemma as described in the previous section are clearly a result of inadequate support for DP among NHS staff, including physicians, nurses, social workers, and possibly other health professionals [12].

\section{Knowledge Management}

Knowledge is a multifaceted concept with multi-layered meanings [12]. Due to this nature, it has become important to manage knowledge in order to drive performance by ensuring that relevant knowledge is delivered "to the relevant person in the right place in a timely fashion" [12] (or simply put right knowledge is delivered to the right person at the right place at the right time). Apart from existing in the human mind (i.e. tacit knowledge), knowledge can exist in physical records (i.e. explicit knowledge), such as patient records and medical notes, which needs to be accessed more readily in paper or in computerised form. The major focus of KM in healthcare is to create environments for "knowledge workers" to develop, leverage and share knowledge. For this to happen effectively KM requires deep-rooted strategic and behavioural change. From this point of view KM represents an evolution towards greater personal and intellectual freedom [21] empowering individuals to engage more actively in their work by sharing ideas, thoughts and experiences [10]. Once knowledge has been discovered, storing it, reusing it and generating new knowledge from it, is important to "adding value" to data to create shared knowledge.

Continued progress in technology makes sharing knowledge easier, and the Internet with collective portals makes knowledge accessible to a wider range of audience [21]. The rise of networked computers has made it easier and cheaper to codify, store and share knowledge [22]. There is no shortage of technologies to aid in managing knowledge in a healthcare environment [10]; rather the prevalence of such technologies can create confusion. The goal of KM is to enhance the performance of a process (e.g. DP) by providing efficient access to knowledge and communities of healthcare professionals. It aims to combine the information from different sources (tacit, implicit and explicit) and provide it on a platform which allows applications to be built on it. It aims to prioritise, share, consolidate and provide consistent and accurate information and performance indicators to help with efficient decision making processes. As workers in a "knowledge intensive environment", healthcare professionals inevitably hold a considerable amount of experiential knowledge, which can be used to solve day to day problems related to decisions on patient discharge. It is important that knowledge used to solve such problems is captured, shared and reused in order to prevent the lack of "nourishment" (i.e. update and replenishment) of that knowledge [23] and to improve "knowledge of context". The "knowledge process" in a healthcare environment can be used to increase collaboration among clinicians, 
nursing staff with social service agencies for purposes of innovation and process improvement. Updating of knowledge assets cultivates the collective knowledge in a healthcare environment, enriching effective management, smoothening the flow of knowledge to enable better problem solving $[23,24]$ and increase "knowledge potential".

KM when applied effectively can result in increased efficiency, responsiveness, competency and innovation [6] which results in superior performance in potentially critical applications [5] such as patient discharge. The challenge is therefore to create a KM system that can acquire, conserve, organise, retrieve, display and distribute what is available in a manner that informs, educates and facilitates the discovery of new knowledge to contribute [9] to the benefit of the organisations. KM can therefore be viewed as an integrator that offers a framework for balancing the technologies and approaches to provide valuable decision making actions [25]. It integrates into a seamless whole by aligning organisational information and practices with the organisation's objectives. This fits into an employee's daily work activities, manages content effectively, and encourages the potential opportunities of knowledge sharing with external agents [6].

\section{Knowledge Management in Healthcare}

The healthcare industry has been called "data rich while knowledge poor" [24] as its functions hold large amounts of data (e.g. patient records, outcomes of surgery and medical procedures, clinical trial data etc.) and yet the knowledge potential of many actions is yet to be fully exploited because much of the data is not translated into knowledge (i.e. there is low added value) in order to provide a wider context, a deeper understanding and to help with strategic decision making [26]. Knowledge appears to be underutilised at the point of care and need [26]. This limits the ability of experienced personnel to "harvest" knowledge and provide a clearer understanding of the involved process and factors by providing "a window on the internal dynamics of the healthcare enterprise" [27]. Multidisciplinary healthcare teams harvest personal expertise essential for patient safety, learn from it, adapt it to local situations and individual patients, then distribute it via reliable networks to care givers to improve care quality. The healthcare application with a "high value form of information" that allows sharing of the lessons learned from past experiences [27] (e.g. knowing what factors to take into account when planning the discharge of a patient) improves the context of knowledge potential processes in future.

A fundamental challenge faced by clinical practitioners and healthcare institutions is in the ability to inter- pret clinical information to make potentially lifesaving decisions while dealing with large amounts of data [10]. Clinical practise is quantitative and very much qualitative too. The tacit knowledge acquired by clinicians and nurses over the years, mainly through experience represents a valuable form of clinical knowledge [19]. KM in Healthcare involves understanding diseases, hospital systems and most importantly patients [19]. [9] argues that clinical methods exist for understanding diseases and illnesses but clinical methods or models are not so readily available for understanding patients. When quantitative and qualitative methods complement each other, and when various modalities of knowledge are used, a holistic view of a situation is best obtained thus leading to efficient decision making [9]. KM strategies can be broadly classified into codification (where knowledge is identified, captured, indexed and made available) and personalisation, where tacit knowledge is shared by means of discussion, effective communication through a multidisciplinary approach, allowing for creative problem solving [9]. In Healthcare, the use of both strategies of understanding diseases and patients is according to the different scenarios. When dealing with routine cases, the codification strategy can be applied and when dealing with a situation where a more creative solution is required, the personalisation strategy can be applied [9]. This approach, however, usually only works when the required knowledge is shared (i.e. processed) successfully. For example the National Institute for Clinical Excellence (NICE), in framing its guidelines, has noted a lack of willingness to share knowledge on the part of doctors who could potentially contribute to the guidance it gives [28].

\section{Knowledge Management for Discharge Planning}

A hospital is a dynamic environment, with changes taking place rapidly as patients move from one ward to another and treatments are carried out over time. Similarly, DP involves changes from a stable temporal state to another with an element of unpredictability of what is going to happen next [23]. In this context the past experiential knowledge of doctors and nurses is useful in assessing situations and deciding on plans. This enables making critical decisions, as their knowledge can be reconfigured and extended to fit the new situation and provide a personalised approach in assessing patients' journey along codified guidelines [23]. KM may have the potential to remove bottlenecks to improve the DP process mapping and identify possible improvement opportunities [12]. Understanding the relevant knowledge for a given situational decision is crucial to this process and a decision can never be completely separated from the context in 
which it is made [6]. This implies that in a hospital setting when looking at DP the interrelated factors need to be considered in the context of knowledge process [12]. Clearly, monitoring and understanding a patient's condition after discharge is a key part of successful DP and this requires the support of appropriate sensing and monitoring technologies.

\section{The Internet of Things}

This section of the paper examines the Internet of Things (IoT) as a possible solution to some of the problems of knowledge sharing in a healthcare environment. The IoT is an abstract concept in which physical objects are identified and connected to each other and have the ability to communicate with and to interact with their surroundings, providing some intelligence in their functionality. "IoT" is concerned with the network of smart embedded devices that communicate with each other and with cloud based applications in order to provide a better quality of life [29]. The term "IoT" refers to the idea of implementing networked RFID technologies with a view to providing a better quality of life and increasing knowledge sharing [29]. The implementation of the IoT may drive advancements in all areas of human life by extracting knowledge from the raw data provided, with the possibilities of solving many socio-technical interaction problems. [30] goes on to discuss the benefits and applications that have already been made by similar systems and suggests that the function of the IoT is to make tasks simpler, quicker and more efficient, providing results to a higher quality than is possible with purely human intervention.

The aim of the IoT in relation to healthcare is therefore to create a better quality of life by connecting "Things", which can be people (e.g. patients and medical staff), objects (e.g. medical equipment) and systems (e.g. patient monitoring systems) and integrating them seamlessly into network using a world-wide web (WWW) technology and connectivity [31]. For instance, healthcare systems could remotely monitor the vital signs of a patient and homes could be re-designed to help with the complex lifestyles many now live by recognising the preferences of its inhabitants. This information could be remotely monitored after patient discharge and the consequent improvement in home care would reduce the incidence of readmission. The integration, evolution and adaptation of emerging biomedical technologies will therefore provide a basic foundation for the IoT [31]. In 2008, the number of Things connected to the Internet already exceeded the number of people on earth, and it is possible that 50 billion Things will be interconnected by the year 2020. A technological evolution of tele-medicine has taken place in which healthcare professionals can now monitor a patient's vital signs remotely and continuously. In addition, the technology supports personalisation, in which patients with chronic conditions are able to live independently in their own homes or secure housing (i.e. a non-hospital setting) using IoT technology to support their lifestyles. Such devices include Lifeline Home Units, Personal Pendants, Wandering Client Alarms, PIR Movement Detectors, Fall Detectors, Bed Occupancy Sensors, Temperature Extreme Sensors, Automatic Medicine/Pill Reminders and Dispensers, Talking Colour Detectors for blind and partially-sighted people and assisted GPS/GSM technologies which recognise when the user goes outside the chosen safety zone [32]. The number of such devices and their applications is increasing daily [32].

\subsection{An Analogy of IoT}

To better understand and explain the IoT concept, [33] uses the human body as an analogy for the IoT, describing parts to sense, connect and control the networks. The "brain" is necessary to recall, process and to provide intelligence to react to the data it receives. The "nervous system", provides a network of sensor signals to and from the "brain", allowing interaction with other parts of the network. "Senses and muscles" provide raw data to the "brain" to understand and react to, allowing response to its environment. By considering the "brain" to be the servers for processing, the "nervous system" to be the communication networks and the "senses and muscles" to be instruments for data capture and actuation, it is understandable how these systems could be considered "intelligent".

\subsection{The Senses \& Muscles}

Machine-to-Machine communications (M2M) is a domain of computing which is essential for the development of the IoT. It deals directly with the physical objects and provides a means of capturing and returning data to a central controller for processing, analysis, correlation and reaction, which may include alerting users, changing attributes of a Thing or communicating with another system. The major difference between the M2M and IoT concepts concerns the "interconnectedness" of their supporting systems. The IoT concept suggests a ubiquitous ecosystem made up of a matrix of services and sub-systems working collectively, whereas M2M systems simply represent sub-systems of the IoT. Protocols for IoT communications, processing and storage are vital, all of which must be highly scalable, versatile and adaptable for changing environments and requirements. The purpose is to provide an entire system (from sensor to software) to capture, analyse and act upon information passing among Things in the network. Various sensors can be deployed, depending on the requirements and the 
attributes that require monitoring.

Sensors and actuators require interfaces to the network infrastructure, known as a gateway, which manages connections to and from the base station, sending the digital values to be processed by control systems. Gateways deal with initial data processing, validation, caching, remote management of devices and security. They must be configured to understand the specific protocol of each sensor, which often causes issues due to the lack of standards between sensors and protocols. One of the biggest difficulties for M2M systems currently is developing an open standardised framework for sensors to connect to gateways allowing mobility and flexibility between systems and ultimately supporting the heterogeneity for IoT. With sensors and actuators connected to a network interface, monitoring applications can be developed to operate in domestic and medical environments [34].

Although sensors, actuators and gateways can be combined in to one unit they are often separated and connected via wired or wireless technologies.

Sensors, actuators and gateways can be deployed in various styles to maximise scalability, utilisation and manageability, including star, mesh, bus and other common network topologies. To deal with communications between sensor-to-sensor, sensor-to-gateway and gateway-to-network various methods are used, including Ethernet, 3G, 4G, satellite communications, Wi-Fi, ZigBee, etc. A basic requirement for the IoT is mobility and flexibility of Things and to achieve this Wireless Sensor Networks (WSN) are designed to allow flexibility and redundancy. In order to manage devices remotely, to handle various protocols and to control low level architectures, embedded frameworks allow developers to focus on functional logic rather than protocol specifics which speeds deployment. If open standards are not adopted progress in M2M and IoT systems will be limited, so to provide communications between gateways and base stations a client/server paradigm is most commonly used. Hyper Text Transfer Protocol (HTTP) drives the Internet and IoT gateways use HTTP to make requests and receive data, although for this purpose HTTP is considered to be extremely demanding, requiring high overheads to maintain and ensure efficient communications.

\subsection{The Nervous System}

For M2M systems to function and expand a reliable open network is a major requirement. The Internet provides a suitable backbone for transferring data anywhere and between virtually anything. Both Cloud Computing and M2M will evolve into components supporting the IoT. To provide an accessible universal network the Internet, or the "Cloud" as it has aptly been named, is evolving into such a network. This will provide a suitable solution meeting the requirements of the IoT. The Cloud can support connectivity anywhere, between anything, at any time and adapt to its environment [35]. The cloud is essentially a modern day metaphor for the efficient use of the internet, the next stage in the internet's evolution, encourage resource optimisation to develop flexible services consisting of remote assets lowering responsibility and cost for the user. Cloud computing refers specifically to the architecture that supports the Cloud. That is the hardware, networks, hypervisors, operating systems, middleware and databases which software and applications rely on. Receiving them as a service reduces management, maintenance and investment. The need for private infrastructure, software licencing, training and many other costly responsibilities are less of a burden compared to traditional "on-premise" solutions. Cloud service providers generally hold all liability, but public infrastructure must be taken into account due to the associated risks with regards to security [35]. Compared to traditional models for distributed applications which often vary in type, style, complexity and interoperability, cloud services provide a cost effective, adaptable and reusable way of deploying services [36].

By optimising existing systems to provide services capable of multi-tenancy supporting adaptability, redundancy, transparency and ubiquitous functionality, which has become a major driving force for Cloud Computing. These characteristics allow quality systems to be developed with minimal resources and investment required, in turn reducing IT costs by maximising hardware utilisation, efficiently provisioning resources and improving user experience [37]. By consolidating resource pools, costs are reduced by between $50 \%-70 \%$ due to improved management, control and utilisation. VMware also states that return on investment is met in less than one year providing profits fast. A study by $\mathrm{PwC}$ also shows that the most important use of the cloud is to deploy IT solutions faster than in-house options by utilising external service providers and lowering costs due to the superior technical skills required to satisfy new requirements.

\subsection{The Brain}

To assist in deploying, managing and controlling remote devices various management and host functions would be required to deal with the vast amounts of data and connectivity to and from other systems. Typical M2M systems specifically require a base station with the following components:

- Databases for storage, retrieval and access;

- API functionality to provide logic and to develop custom programs for reusability, connectivity and 
data access;

- Web services to provide access to API's via the internet to again reuse business logic and maximise use;

- Web applications providing business logic and functionality dealing with data input, processing and output;

- Interfaces for user access which provide enhanced interaction, control, flexibility and feedback;

- Flexible programming languages are required to retrieve information and allow access to the system. Lightweight and efficient protocols are preferred to minimise bandwidth and speed software development;

These components provide the tools to develop and manage M2M systems. By suitably deploying each component data can be stored appropriately and communicated via a number of different methods which supports openness by allowing numerous interfaces for connectivity. This is essentially the "brains" of an M2M system providing logic and control. To counterpart the base station of an M2M system, deploying embedded devices integrated into Things can provide a suitable way for constant and reliable services, access and enhanced functionality, especially when network connections are limited. Pervasive computing concerns the integration of micro-computers in a less obvious fashion, to be indistinguishable in everyday tasks operating without human interaction. The aims of pervasive computing are to improve human experiences and quality of life by implementing technologies in a less evasive way [38]. The impact of pervasive computing on M2M technologies can allow for complex computational programs on smaller and less demanding devices, such as are suitable for comfortable patient monitoring in an aftercare situation. This allows for sensors and gateway devices to be enhanced, adding additional functionality and reducing cost. When combined with the Cloud and M2M technologies, applications allow for pervasive systems to infuse stationary and passive objects with mobility and dynamism allowing them to act and react to their environment, ultimately providing the self-aware environment for the IoT [38].

By empowering inanimate objects with sensors, actuators, computing power and a communication interface it allows the object to become an interactive part of the environment in which it resides. Embedding such technology into an object allows for programs to run on the object and for complex systems to manage and analyse objects in a group. Common characteristics of pervasive computing include heterogeneity, automation, transparency, mobility, scalability and security, all of which align with the aims of the IoT. The IoT will depend on pervasive computing in order to empower, control and manage the sensor and actuator networks and to provide enhanced interfaces to the cloud. The ability of a device to react to its environment suggests it possesses intelligent characteristics which have produced the term "Smart device", referring to a device with a range of functional capabilities allowing it to react to other smart devices or systems. To enhance mobility and lower evasiveness objects may connect to more powerful devices in their surroundings, providing the same smart nature without the resources embedded in an object [39]. In isolation these devices may not be considered "smart" as the same tasks which depend on the infrastructure and communications provided by more powerful devices [39]. "Intelligent environments" describes a group of smart devices communicate in order to benefit the user. This is a step towards the IoT and a major achievement for pervasive computing. Personal Area Networks (PAN) provides methods of interlinking such devices to react to data sources specified by user.

The term "smart" became prevalent due to "smart phones", referring to mobile telephones with features allowing them to react to data automatically. Smart devices allow for custom software to be installed and allow data to be stored, processed and sent to network services without user interaction. RFID, barcode and other similar identifying technologies require dedicated systems to instil intelligence therefore it cannot be a smart device. Other authors also argue that smart devices should be capable of acquiring and applying knowledge autonomously and automatically to its environment, adapting to inhabitants' preferences and requirements [40]. Smart devices can be categorised by size, form factor, technology and purpose. [41] classifies six types including tabs, pads, boards, dust, skin and clay. Each device can also be classified as passive or active. Passive refers to relying on infrastructure and on-object memory such as barcodes or passive RFID. Active devices typically consist of computational power and do not rely completely on sensor networks and applications to hold state information. A repeating theme with regards to $\mathrm{M} 2 \mathrm{M}$, Cloud and the IoT is taxonomy and RTLS (Real time Location-based Services) which allow the physical location of an object to be communicated (e.g. the location of a nurse or doctor within a hospital or an at-risk patient within their home. [42] describes how the following methods for RTLS may be implemented:

- Geographical-Using spatial information to map location;

- Special-Identify the item by the size of its surrounding;

- Proximity - Detect distance from other devices and sensors;

- Transitional-Recognised by referenced fixed receivers. 
This is a vital component for environments to react as the presence of an object or device which can imply that a human has entered a room, an object has left a specific zone or a new device can be utilised in an environment. Trends in pervasive computing have focused on miniaturisation and embedded frameworks to support web services, APIs and artificial intelligence. Artificial intelligence would allow a device, or environment, to adapt and learn a hosts habits and preferences to customise operations to their needs. Similarly this logic relates to the current use of internet data being tailor for targeted advertising and product services to the user [43]. In a healthcare setting therefore, as vast amounts of data and information is generated, the ability of "smart" devices to feed knowledge back into the system to help make more informed and personalised decisions with regards to DP and other areas would be of significant importance.

\subsection{Conclusion}

This paper has examined the problem of patient readmission into hospitals. It has suggested ways of reducing the number of patient readmissions through improved DP. The study suggests that KM may be a way of reducing readmissions and improving DP through improved knowledge of the patient's aftercare environment and medical situation. The Internet of Things (IoT) is suggested as a way of monitoring patients continuously and effectively once they have left hospital (e.g. in their own homes after discharge or in other healthcare situations).

The IoT is discussed in this paper because it is a candidate technology which will enforce knowledge sharing. The Smart environment will allow information to be generated by Things and will automatically been sent and stored in a cloud. Through the use of Artificial Intelligence techniques, data will be analysed and categorised accordingly. The role of KM is to use this information to inform the design of DP upon a patient's admission in hospital. The proposed KM Model will consider factors, internal to a healthcare setting, external to a healthcare setting and psychosocial factors of patients in order to make informed decisions, thus resulting in effective and personalised DP.

\section{REFERENCES}

[1] NHS, "About the NHS," 2012.

http://www.nhs.uk/NHSEngland/thenhs/about/Pages/over view.aspx

[2] P. Ashmos and P. Huber, "The Systems Paradigm in Organization Theory: Correcting the Record and Suggesting the Future," JSTOR: The Academy of Management Review, Vol. 12, No. 4, 1987, pp. 607-621. http://jaylee.business.ku.edu/MGMT916/PDF/AshmosHu ber 1987 AMR.pdf

[3] L. Ervin, "The Systems View of the World," Hampton
Press, New Jersey, 1996

[4] A. D. Chetty, V. K. Kartha, A. McKenna, K. DePaoli, M. R. B. Jack, "Re-Engineering the Hospital Discharge: An Example of a Multifaceted Process Evaluation," Advances in Patient Safety: From Research to Implementation, Vol. 1-4, Agency for Healthcare Research and Quality, Rockville, 2005. http://www.ahrq.gov/qual/advances/

[5] I. Reychav and J. Weisberg, "Bridging Intention and Behaviour of Knowledge Sharing," Journal of Knowledge Management, Vol. 14, No. 2, 2010, pp. 285-300.

http://www.emeraldinsight.com/10.1108/1367327101103 2418.

[6] M. Fontaine and E. Lesser, "Challenges in Managing Organizational Knowledge," 2002.

http://www-935.ibm.com/services/us/imc/pdf/g510-323400 -esr-managing-organizational-knowledge.pdf

[7] A. Hannan, "Opportunities and Challenges of Using Electronic Health Records to Enhance Patient Care," 2010. http://www.bcs.org/upload/pdf/assist-140910-ahannan.pdf

[8] M. Polanyi, "Tacit Knowing," In: M. Polanyi, Ed., The Tacit Dimension, Doubleday \& Company, New York, 1966, pp. 3-25.

[9] H. J. Levenstein, C. E. McCracken, M. R. Ian, A. M. Stewart and B. J. Brown, "The Patient-Centred Clinical Method," Family Practice, Vol. 3, No. 1, 1986, pp. 24-30. http://fampra.oxfordjournals.org/content/3/1/24.full.pdf $+\mathrm{h}$ tml

[10] A. Eardley and A. Czerwinski, "Knowledge Management for Primary Healthcare Services," In: R. Bali and A. Dwivedi, Eds., Knowledge Management: Issues, Advances and Successes, Springer Science + Business Media, New York, 2007.

[11] R. K. Bali, A. Dwivedi, A. E. James, R. N. G. Naguib and D. Johnston, "Towards a Holistic Knowledge Management Framework for Healthcare Institutions," Proceedings of the Second Joint EMBSBMES Conference, Houston, 2002, pp. 1894-1895.

http://ieeexplore.ieee.org/stamp/stamp.jsp?arnumber $=010$ 53081

[12] N. Kamalanathan, A. Eardley, C. Chibelushi and P. Kingston, "The Discharge Planning Dilemma in the UK NHS: The Role of Knowledge Management," In: G. Huang, et al., Ed., Health Informations Systems, Springer-Verlag, Berlin Heidelberg, 2013, pp. 172-185.

[13] Sg2 Healthcare Intelligence, "Reducing 30-Day Emergency Readmissions," 2011, pp. 1-28.

http://www.hsj.co.uk/Journals/2/Files/2011/6/15/Sg2_Ser vice Kit_Reducing30-DayReadmissions.pdf

[14] K. Ishikawa, "Introduction to Quality Control," 3A Corporation, Tokyo, 1990.

[15] Social Care Institute for Excellence, "SCIE Research Briefing 12: Involving Individual Older Patients and Their Carers in the Discharge Process from Acute to Community Care: Implications for Intermediate Care." http://www.scie.org.uk/publications/briefings/briefing 12

[16] Institute for Healthcare Improvement, "Improving Patient Flow at Bon Secours Venice Hospital," 2011.

http://www.ihi.org/knowledge/Pages/ImprovementStories 
/ImprovingPatientFlowBonSecoursVeniceHospital.aspx

[17] N. Triggle, "Organise Urgent Care Better, NHS Told," BBC News, 2012.

http://www.bbc.co.uk/news/health-19181905

[18] R. Ramesh, "One in Four Hospital Patients Could Be Recovering at Home," The Guardian, 2011. http://www.guardian.co.uk/society/2011/dec/29/hospitalpatients-discharge-bed-blocking

[19] S. Adams, “'Intolerable' Bed-Blocking Crisis Threatens NHS," The Telegraph, 2011.

http://www.telegraph.co.uk/health/healthnews/8343879/I ntolerable-bed-blocking-crisis-threatens-NHS.html

[20] R. Winnett, 'Scandal of NHS 'Production Line' as Readmissions Soar," The Telegraph, 2011. http:/www.telegraph.co.uk/health/healthnews/8983505/S candal-of-NHS-production-line-as-readmissions-soar.html

[21] T. Chunsheng, "The Research on Knowledge Management and Knowledge Portal Framework Based on Agent Mechanism," IEEE (Vol. 00, pp. 263-267), Hefei, China. 3rd World Congress on Intelligent Control and Automation.

http://ieeexplore.ieee.org/stamp/stamp.jsp?tp=\&arnumber $=859962$

[22] T. M. Hansen, N. Nohria and T. Tierney, "What's Your Strategy for Managing Knowledge?" Harvard Business Review, 1999, pp. 1-11.

http://consulting-ideas.com/wp-content/uploads/Whats-yo ur-strat-art.pdf

[23] S. Liao, "Problem Solving and Knowledge Inertia," Expert Systems with Applications, Vol. 22, No. 1, 2002, pp. 21-31.

http://inkinghub.elsevier.com/retrieve/pii/S09574174010 0046X

[24] A. M. Mills and T. A. Smith, "Knowledge Management and Organizational Performance: A Decomposed View," Journal of Knowledge Management, Vol. 15, No. 1, 2011, pp. 156-171.

http://www.emeraldinsight.com/10.1108/1367327111110 8756

[25] P. J. Sharp, W. A. Eardley and H. Shah, "Visual Tools within MakE: A Knowledge Management Method, Electronic," Journal of Knowledge Management, Vol. 1, No. 2, 2003, pp. 177-186.

[26] A. Copper, "We Haven't Got a Plan, So What Can Go Wrong? Where Is the NHS Coming from?" In: R. K. Bali and A. Dwivedi, Eds., Healthcare Knowledge Management, Springer Science + Business Media, New York, 2007, pp. 221-232.

[27] S. Shepperd, J. McClaran, C. Phillips, N. Lannin, L. Clemson, A. McCluskey, I. Cameron and S. Barras, "Discharge Planning from Hospital to Home," The Cochrane Library, Vol. 1, 2010, pp. 1-75.

http://onlinelibrary.wiley.com/doi/10.1002/14651858.CD 000313.pub3/pdf

[28] NHS, "Leaving Hospital," 2012 http://www.nhs.uk/nhsengland/aboutnhsservices/nhshospi tals/pages/leaving-hospital.aspx
[29] Theinternetofthings.eu, "Russian Research Center on the Internet of Things Is Official Partner for the Internet of Thing Council: The Internet of Things."

http://theinternetofthings.eu/russian-research-center-inter net-things-official-partner-internet-thing-council

[30] D. Evans, "The Internet of Things: How the Next Evolution of the Internet Is Changing Everything," 2012. http://www.cisco.com/web/about/ac79/docs/innov/IoT IB SG_0411FINAL.pdf

[31] C. Pfister, "Getting Started with the Internet of Things," O’Reilly Media, Inc., Sebastapool, 2011.

[32] M. R. McGee-Lennon and P. D. Gray, "Including Stakeholders in the Design of Home Care Systems: Identification and Categorization of Complex User Requirements," INCLUDE Conference, London, April 2007.

[33] H. Ning, "Future Internet of Things Architecture: Like Mankind Neural System or Social Organization Framework?" IEEE Communications Letters, Vol. 15, No. 4, 2011, pp. 461-463.

[34] I. F. Akyilidiz, T. Melodia and K. Chowdhury, "A Survey on Wireless Multimedia Sensor Networks," Journal of Computer Networks, Vol. 51, No. 4, 2007, pp. 921-960. doi:10.1016/j.comnet.2006.10.002

[35] J. Hurwitz, "Cloud Computing for Dummies," Wiley Publication. Hoboken, 2010.

[36] Exelanz, "Cloud vs. Traditional Hosting," 2012. http://www.exelanz.com/cloud-vs-traditional-hosting/

[37] Fasthosts, "The Myth behind the Extinction of IT Departments," 2012.

http://blogs.fasthosts.co.uk/cloud-computing/the-myth-be hind-the-extinction-of-it-departments/?goback $=\% 2$ Egde 45151_member_191264989

[38] D. B. Lange and M. Oshimma, "Ten Good Reasons for Mobile Agents," Communications of the ACM, Vol. 42, No. 3, 1998, pp. 88-89. doi:10.1145/295685.298136

[39] M. Satyanarayanan, "Pervasive Computing: Vision and Challenges," IEEE Personal Communications, Vol. 8, No. 4, 2001, pp. 10-17. http://www.cs.cmu.edu/ aura/docdir/pcs01.pdf

[40] D. J. Cook, M. M. Youngblood, E. O. Heiermann, K. Gopalratnam, S. Rao, A. Litvin and F. Khawaja, "Mavhome: An Agent-Bqased Smart Home," Proceedings of the First IEEE International Conference on Pervasive Computing and Communications, Fort Worth, 26 March 2003, pp. 521-524.

[41] S. Poslad, "Ubiquitous Computing: Smart Devices, Environments and Interactions," John Wiley and Sons, London, 2011.

[42] K. M. Dombroviak and R. Ramnath, "A Taxonomy of Mobile and Pervasive Applications," Proceedings of the 2007 ACM Symposium on Applied Computing, Seoul, 11-15 March 2007, pp. 1609-1615.

[43] D. Chaffey, "Internet Marketing: Strategy, Implementation, and Practice," Financial Times, Prentice Hall, Harlow, 2000. 